\title{
The Novel Dimensions of Cardio-Metabolic Health: Gut Microbiota, Dysbiosis and its Fallouts
}

\author{
Vinod Nikhra* \\ Department of Medicine, NDMC Medical College, India
}

Submission: February 02, 2019; Published: June 21, 2019

*Corresponding author: Vinod Nikhra, Senior Chief Medical Officer and Consultant, Department of Medicine, Hindu Rao Hospital and NDMC Medical College, New Delhi, India

\begin{abstract}
The New Dimension in Cardio-Metabolic Health: Cardio-metabolic disorders (CMDs) encompass various disorders like metabolic syndrome, obesity, diabetes and cardiovascular disease (CVD) including atherosclerosis, hypertension, coronary artery diseases, cerebrovascular disease and heart failure. CMD occur due to insulin resistance (IR), inflammation, deranged energy and metabolic homeostasis. Apart from various predisposing factors including genetic and environmental and lifestyle factors, the novel factors such as nutritional and gut microbial dysbiosis have also been implicated for CMDs.
\end{abstract}

Microbial Dysbiosis and Metabolic Disorders: Gut microbiota exerts a significant role in the pathogenesis of the metabolic syndrome and related diseases. The impairment of the fine balance between gut microbes and host's immune system culminates into intestinal microbial dysbiosis and development of metabolic endotoxemia, leading to systemic inflammation and IR. There has been outlined the gut microbiotadependent mechanisms linking the gut microbial dysbiosis and the development of metabolic disorders like insulin resistance, diabetes, obesity and non-alcoholic fatty liver disease (NAFLD).

Microbial Dysbiosis and Atherosclerosis: The recent clinical and animal studies have documented that gut dysbiosis contributes to the development of atherosclerosis through modulation of various pathways. There is evidence that gut microbiota plays a causative role in atherosclerosis by modulating inflammation through production of microbial metabolites like short-chain fatty acids (SCFAs). The secondary bile acids are another group of gut microbiota-derived metabolites involved in CVD and various metabolic diseases.

Microbial Dysbiosis and Hypertension: Apart from, dyslipidemia, hypertension is another most important risk factor for CVD that bears genetic susceptibility and influenced by environmental factors. The gut dysbiosis has been linked with cerebrovascular events apart from metabolic disorders. Simultaneously, there exists a relationship between gut microbiota and hypertension, which has been established by various recent studies. Both SCFAs and oxidized LDL have been associated with the microbiota-linked mechanisms for development or exacerbation of hypertension.

Microbiota-Targeted Therapy for CMD: The gut microbiota is a novel therapeutic target for the treatment of CMD. Over the recent years, there have evolved potential strategies for therapeutically targeting intestinal microbiota and microbial processes. The MTT appears to be a promising strategy to prevent and treat metabolic disorders as well as CVD. The most frequently used measures to manipulate the gut microbiota to reduce CV risk and CVD are therapeutic use of probiotics, prebiotics and certain nutraceuticals. The therapeutic intervention to repair microbial dysbiosis through fecal microbiota transplantation (FMT) appears to be a potential therapy for CVD and various metabolic disorders like metabolic syndrome, T2DM and obesity.

Keywords: Atherosclerosis; Dysbiosis; FMT; Hypertension; Insulin resistance; Metabolic syndrome; Microbiota; Microbiota-targeted therapy; NAFLD; Obesity; Prebiotics; Probiotics; Resveratrol

Abbreviations: CMDs: Cardio-Metabolic Disorders; CVD: Cardiovascular Disease; IR: Insulin Resistance; NAFLD: Non-Alcoholic Fatty Liver Disease; SCFAs: Short-Chain Fatty Acids; FMT: Fecal Microbiota Transplantation; MetS: Metabolic Syndrome; HF: High-Fat; CONV-R: Conventionally Raised Mice; GLP-1: Glucagon-Like Peptide-1; LPS: Lipopolysaccharides; TLR-4: Toll-like Receptor 4; NASH: Non-Alcoholic Steatohepatitis; PAMPs: Pathogen Associated Molecular Patterns; NF-кB: Nuclear Factor Kappa B; TNF- $\alpha$ : Tumor Necrosis Factor-Alpha; PG: Peptidoglycan; NOD: Nucleotide-Binding Oligomerization Domain; PRRs: Pattern Recognition Receptors; TMAO: Tri-Methyl-Amine N-Oxide; TMA: Trimethylamine; FMO: Flavin Monooxygenase; CA: Cholic Acid; CDCA: Chenodeoxycholic Acid; DCA: Deoxycholic Acid; LCA: Lithocholic Acid; BSH: Bile-Salt Hydrolase; MOS: Mannan Oligosaccharide; FDA: Food and Drug Administration

\section{The New Dimension in Cardio-Metabolic Health}

The human gut microbiota comprises of a diverse ecosystem of over 1014 microorganisms, having over 1100 diverse species, residing in the gastrointestinal tract. It is mainly composed of
Bacteroidetes, Firmicutes, Actinobacteria, Proteobacteria and Cerrucomicrobia species, of which the first two, namely Bacteroidetes and Firmicutes account for $\sim 90$ percent of the overall gut 


\section{Current Research in Diabetes \& Obesity Journal}

microbiota [1]. The microbiota exists in a symbiotic relationship with the host and plays a critical role in maintaining metabolic homeostasis. It has been established that the homeostasis of gut microbiota is critical for maintaining human health in general as well as CV health [2]. Further, the alterations in gut microbiota can points to the latent CVD as well as have a prospective value [3]. The gut dysbiosis denoting loss of microbial diversity and imbalance, accompanied with reduced intestinal integrity, has been related to the development of various diseases including CVD, obesity [4,5] T2DM [6,7] NAFLD [8,9] and various malignancies $[10,11]$ (Figure 1).

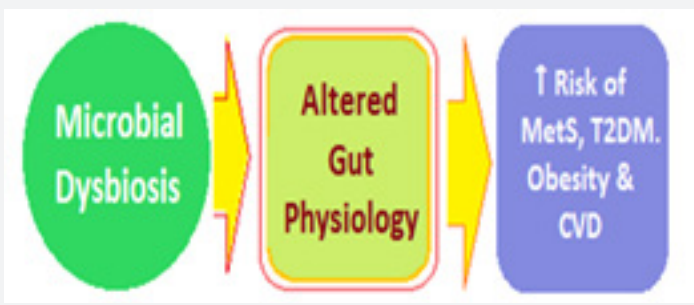

Figure 1: Microbial dysbiosis and its metabolic and cardiovascular fallouts.

The microbiota plays a role in various metabolic and physiological functions, like modulation of regulation of appetite, satiety and energy equilibrium, glucose and lipid homeostasis, production of vitamins and metabolites having multiple actions by regulation of several biochemical and physiological mechanisms. The abnormal alteration in the gut microbiota composition called dysbiosis can lead to several diseases, including metabolic disorders, such as metabolic syndrome, diabetes, obesity and cardiovascular diseases. This is due to IR, inflammation, deranged homeostasis and metabolic disorders, vascular disorders like atherosclerosis, hypertension, coronary artery diseases, cerebrovascular disease and heart failure [12]. Thus, apart from various predisposing factors including genetic and environmental and lifestyle factors the novel factors such as nutrition and gut microbial dysbiosis have also been implicated as the main risk factors for metabolic syndrome (MetS), obesity, diabetes and CVD. The consumption of a high-fat (HF) diet has been shown to induce gut dysbiosis [13]. There has been outlined the gut microbiota-dependent mechanisms of CVD, highlighting the relationship between gut microbial dysbiosis and the development of atherosclerosis and hypertension, both being major risk factors for CVD [14].

\section{Gut Microbial Dysbiosis and Metabolic Syndrome}

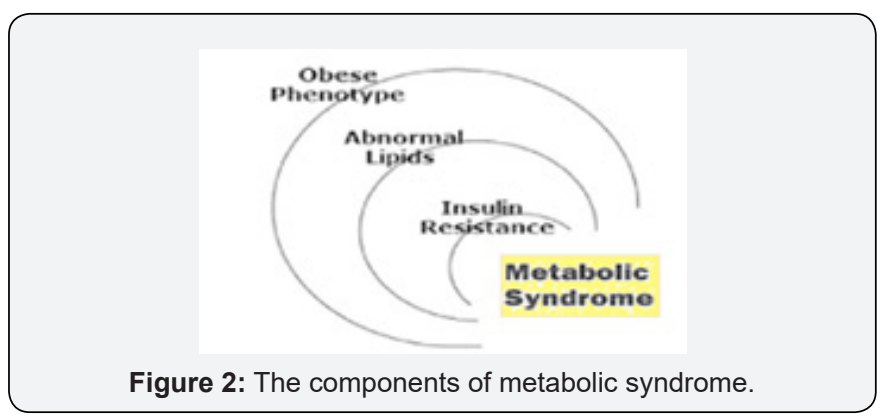

The MetS is a combination of interconnected physiological, biochemical, clinical and metabolic factors that has been linked to an increased risk of cardio-metabolic disorders and T2DM. The raised blood pressure, dyslipidemia, raised fasting glucose, central obesity and non-alcoholic fatty liver disease (NAFLD) are the main features of MetS (Figure 2).

\section{Dysbiosis and obesity}

The role of gut microbiota on the development of obesity was proved by studies conducted on germ free mice (GF-mice) compared to conventionally raised mice (CONV-R) [15]. The CONV-R have a $40 \%$ higher body fat content than GF-mice, a phenomenon independent from the food intake. Moreover, after colonization of GF-mice with intestinal flora coming from CONV-R mice, a significant increase of body weight and $\sim 60 \%$ increase of body fat, along with increased hepatic triglycerides synthesis and IR in recipients (CONV-D), independent of food intake and total energy expenditure. Further, the GF-mice colonized with intestinal flora from obese mice showed an evident increase in body weight and fat tissue through an increased energy harvest promoted by gut microbiota [16].

The mechanism through which gut microbes contribute to increased energy absorption appears to be through production of short chain fatty acids (SCFAs), resulting from the hydrolysis and the fermentation of dietary polysaccharides. The SCFAs, such as propionate, butyrate and acetate exert complex metabolic actions influencing host appetite, intestinal transit time, energy absorption and energy harvest [17]. SCFAs also increase intestinal absorption of monosaccharides stimulating the expression of sodium/glucose transporter 1. SCFAs also contribute to modulate host appetite and food intake interacting with G-coupled proteins expressed by enteroendocrine cells and promoting the release of glucagon-like peptide-1 (GLP-1) and peptide YY, which influence satiety [18]. In addition, SCFAs influence lipid metabolism by increasing lipogenesis and inhibiting fatty acids oxidation [19].

The studies have proved specific changes in gut microbiota composition in genetically obese mice (ob/ob mice), compared to lean counterparts, showing a $50 \%$ reduction in the abundance of Bacteroidetes and a proportional increase in Firmicutes. These specific changes appear to contribute to the increased SCFAs production and energy harvest observed both in obese mice and in GF-mice colonized with ob/ob mice microbiota [20].

There are other possible mechanisms. The high-fat diet has shown to increase the proportion of Gram-negative species in gut microbiota, thus, contributing to an increased intestinal absorption of bacterial fragments, such as lipopolysaccharides (LPS). The increased levels of circulating LPS lead to 'metabolic endotoxemia', manifesting as weight gain, fasting hyperglycemia and hyperinsulinemia [21]. There is increasing evidence to suggest that high-fat diet promotes changes in gut microbiota composition, but the subsequent development of obese phenotype has been related to metabolic endotoxemia [22]. 


\section{Current Research in Diabetes \& Obesity Journal}

The increased intestinal permeability leads to bacterial translocation which in turn causes a low-grade intestinal inflammatory state, resulting from the interaction between luminal bacteria and host's immune system. Bacterial antigens are recognized by specific receptors exposed by intestinal dendritic cells, such as NOD1, CD-14 and Toll-like receptor 4 (TLR-4). The interaction between these receptors and bacterial peptidoglycan or LPS activates mucosal inflammation and bacterial translocation, through the activation of the NF- $\kappa$ B pathway. Another mechanism involved in the regulation of gut homeostasis is the endocannabinoid system. The endocannabinoid receptors expressed in the gut, eCB1, interact with bacterial LPS, modulating gut permeability, LPS translocation and inducing metabolic endotoxemia [23].

The complex interactions affect gut microbial homeostasis and composition, and lead to intestinal dysbiosis, bacterial translocation and the subsequent development of metabolic endotoxemia, which is essential for the development of obese phenotype and IR. The functional changes in gut microbiota result in altered genetic pathways expression and lead to a preferential increase in genes involved in sugar and carbohydrate metabolism in obese subjects. The functional changes in gut microbiota lead to an increased production of SCFAs, with a consequent raised capacity of energy harvest associated to a preferential increase of propionate. The diet has a strong influence on the gut microbial composition.

The high-fat/low-fibre Western diet promotes overgrowth of gram-negative pathogens, with consequent increased intestinal translocation of bacterial LPS interacting with specific TLR receptor and culminating into an inflammatory cascade that precedes the development of insulin resistance, obesity and diabetes. The animal-based diet increases the abundance of bile-tolerant and amino-acids metabolizing microorganisms (Alistipes, Bilophila and Bacteroides) and decreases the levels of Firmicutes which metabolize dietary plant polysaccharides. Whereas, with vegetarian diet, Prevotella species is in abundance having preferential expression of genes involved in starch break-down.

\section{Dysbiosis and T2DM}

The gut microbial dysbiosis promotes LPS-induced metabolic endotoxemia, which is the first step leading to the development of IR. Experimentally LPS infusion in mice studies leads to fasting hyperglycemia and hyperinsulinemia. The administration of a broad-spectrum antibiotic therapy which reduces metabolic endotoxemia and modulates gut microbiota, has been shown to improve glucose tolerance in ob/ob and diet-induced obese and insulin-resistant mice [24].

A recent study has outlined the protective role of the bacterium Akkermansia muciniphila against the development of metabolic diseases [25]. The normalization of $A$. muciniphila abundance leads to an improved metabolic profile and reduced fat-mass, metabolic endotoxemia, adipose tissue inflammation and IR. Further, it leads to increased intestinal levels of endocannabinoids that control inflammation, the gut barrier integrity and gut peptide secretion. The butyrate or conjugated linolenic acid producing bacteria such as Bifidobacteria or Lactobacillus, improve glucose tolerance along with a decrease in endotoxemia, pro-inflammatory cytokines and intestinal permeability [26].

The clinical studies have documented that LPS levels are significantly increased in diabetic subjects, compared to controls, and seem to decrease with the administration of antidiabetic therapy [27]. A longitudinal study has noted that increased levels of blood circulating bacteria are present before the development of diabetes [28]. Further, in diabetic subjects, a moderate degree of gut microbial dysbiosis is associated with a selective increase in opportunistic pathogens and decrease in bacteria producing beneficial metabolites, such as butyrate. The metabolite, butyrate exerts a protective role, enhancing the expression of tight-junction genes, promotes gut barrier function and reduces bacterial translocation [29]. There is a role of metabolic endotoxemia in development of IR and T2DM, leading to progressive development of glucose intolerance and IR.

\section{Dysbiosis and NAFLD}

The gut microbiota influences energy absorption and storage by modulating monosaccharides absorption and hepatic lipogenesis through genes expression pathways involved in complex metabolic reactions. The GF mice receiving gut microbial colonization from conventional mice show a significant increase in triglycerides synthesis and fatty storage in hepatocytes. The study revealed that mice developing IR and fatty liver showed an increased number of Lachnospiraceae and Barnesiella, and decreased Lactobacilli. Further, gut microbiota may contribute to development of fatty liver through the ethanol production, as in the genetically obese mice breath ethanol levels were found to be significantly higher than in the lean mice and antibiotic treatment reduced by $50 \%$ the cumulative ethanol production in the obese mice [30].

Another mechanism through which the gut microbiota influences the susceptibility to develop NAFLD is through alteration of the choline and bile acid metabolisms. Recently, the role of fructose-rich diet has been implicated in NAFLD. The fructose induced-NAFLD is associated with the development of small bowel bacterial overgrowth, increased intestinal permeability, increased circulating endotoxin and subsequent Kupffer cells mediated hepatic inflammation [31]. Gut microbiota also exerts a role in the progression from NAFLD to non-alcoholic steatohepatitis (NASH) and hepatic fibrosis. There is a role of cytoplasmic multiprotein complexes called inflammosomes, in the development of inflammatory hepatic changes. The inflammosomes are expressed in most liver cells, such as Kupffer cells, liver sinusoidal endothelial cells, periportal myofibroblasts and hepatic stellate cells. The activation of cytosolic inflammosomes, induced by the interaction with LPS or other microbial antigens coming from bacteria circulating in the portal system, leads to the expression of the pro-inflammatory cascade and modulates hepatic tissue fibrosis [32].

The clinical studies outline the similar mechanisms to explain the possible role of gut microbiota in the pathogenesis of NAFLD. There is a small bowel bacterial overgrowth and increased intes- 


\section{Current Research in Diabetes \& Obesity Journal}

tinal permeability, associated with choline deficiency, increased circulating levels of ethanol and intestinal overgrowth of ethanol-producing bacteria, such as Enterobacteriaceae and Escherichia coli. The development of NASH is associated with increased systemic inflammation through TLR-4 mediated interaction with circulating pathogen associated molecular patterns (PAMPs) and release of pro-inflammatory cytokines [33].

\section{Gut Microbial Dysbiosis and Atherosclerosis}

The animal studies and clinical trials, both, have documented that gut dysbiosis can contribute to the development of atherosclerosis through modulation of various pathways [34,35]. The gut microbiota plays a causative role in atherosclerosis by modulating inflammation through production of microbial metabolites [36]. There has been demonstrated relatively lower abundance of Roseburia and Eubacterium, and higher abundance of Collinsella in atherosclerotic patients compared to healthy controls [37]. In addition, Akkermansia muciniphila has been found to improve gut barrier functions and exert protective effects against atherosclerosis [38].

\section{Inflammatory Mechanism for Atherosclerosis}

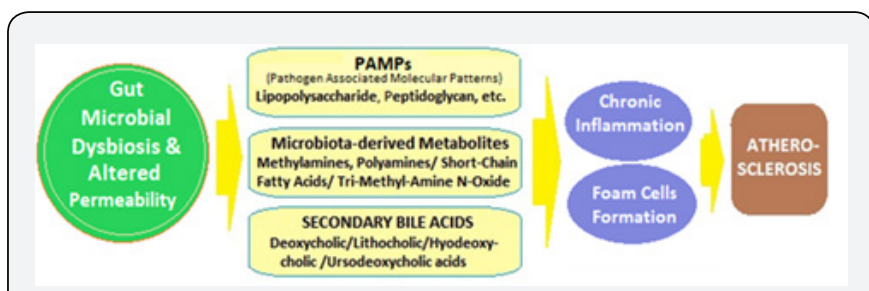

Figure 3: The mechanisms and pathways linking microbial dysbiosis and atherosclerosis.

The gut epithelium is one of the first barriers to protects against the invasion of pathogens. The integrity of the gut barrier is, thus, essential for preservation of health. An abnormal intestinal permeability is due to reduced expression of tight junction proteins, including zonula occludens- 1 , claudin-1, and occludin, and an imbalance between intestinal epithelial cell death and regeneration [39]. With impaired intestinal epithelial barrier, the invasion by PAMPs drives an immune response and results in systemic and tissue-specific inflammation. The impairments to the gut barrier integrity induced by gut dysbiosis, thus, acts as risk factor for chronic inflammation in various diseases including atherosclerosis, which is a chronic inflammatory disease [40] (Figure 3).

Further, the microbial components, lipopolysaccharide (LPS) and peptidoglycan are inflammatory risk factors for CVD. LPS is a cell wall component of Gram-negative bacteria, which has been extensively studied as one of the PAMPs involved in CVD risk. The gut dysbiosis suppresses the expression of tight junction proteins, leading to increased intestinal permeability and the translocation of LPS into the blood [41]. Gut dysbiosis-derived LPS activates the Toll-like receptors (TLRs), which recognize bacterial products and modulate the immune system [42]. The upregulation of
TLRs is associated with inflammatory reactions, which promote atherosclerosis $[43,44]$. The binding of LPS to TLR4 activates the downstream pathways including MYD88 and nuclear factor kappa $B(N F-\kappa B)$, contributing to the increased production of pro-inflammatory cytokines such as IL-6, IL-1, IL-27, and tumor necrosis factor-alpha (TNF- $\alpha$ ), leading to an increased risk of developing CVD $[45,46]$.

Another bacterial PAMP, peptidoglycan (PG), is also associated with CVD risk by impairing the intestinal epithelial barrier. $\mathrm{PG}$ is a minor cell wall component of G-Neg bacteria and a major component of G+ bacteria. The patients with atherosclerosis have shown enrichment of the genes encoding PG synthesis, which is associated with vulnerable plaques in atherosclerotic arteries [47]. Through PG recognition, the nucleotide-binding oligomerization domain (NOD) proteins NOD1 and NOD2 promote intracellular bacteria clearance. The NOD2 being a critical regulator of intestinal bacterial immunity, helps in maintaining integrity of the gut barrier. The potential role of NOD1 in atherosclerosis is proved by studies in Nod1 knockout mice. The knockout of apolipoprotein $\mathrm{E}$ and Nod 1 in mice significantly reduced the development of atherosclerotic lesions [48]. There are other PAMPs promoting inflammatory processes through the engagement of host pattern recognition receptors (PRRs). In addition, the functional changes in the gut microbiota also adversely affect the atherosclerosis risk.

\section{The role of microbial metabolites in atherosclerosis}

Apart from the microbial dysbiosis-associated inflammation, various gut microbiota-derived metabolites also play role in development of atherosclerosis. Various metabolites are produced by the gut microbiota, as well as are product of co-metabolism of gut microbiota, including amines methylamines, polyamines, short-chain fatty acids (SCFAs), tri-methyl-amine N-oxide (TMAO) and secondary bile acids (BAs). SCFAs are critically involved and have impact on various metabolic diseases [49]. TMAO is an important microbial metabolite having various adverse effects and a diet rich in complex starch like potato has been found to increase its plasma levels and the CV risk [50]. Further, the observations from the recent research endorse the involvement TMAO and secondary BAs in atherosclerosis [51].

TMAO and atherosclerosis: In the intestine, dietary phosphatidylcholine or L-carnitine is metabolized by gut microbiota into trimethylamine (TMA), a precursor of TMAO [52]. TMA is transported to liver and oxidized by flavin monooxygenase 3 (FMO3), to form TMAO [53]. In mice studies, the hepatic knockdown of FMO3 by antisense oligonucleotide decreased circulating TMAO levels and attenuated atherosclerosis through activating macrophage reverse cholesterol transport [54]. FMO3, thus appears to be a central regulator of cholesterol balance [55]. The increased plasma levels of gut microbial phosphatidylcholine metabolites and TMAO are associated with the risk of CVD [56,57]. Further, the higher level of plasma TMAO has been correlated with atherosclerotic plaque formation and the extent of the atherosclerotic plaque area, and the risk of heart failure [58]. 


\section{Current Research in Diabetes \& Obesity Journal}

The experimental studies in mice have established that TMAO is a promoter of atherosclerotic disease and an important risk factor for the pathogenesis of CVD. It can be a potential biomarker for CVD risk $[59,60]$. TMAO has been shown to enhance platelet hyperreactivity and thrombosis risk [61]. No wonder, the metabolite, TMAO is apparently a potential therapeutic target for CVD as well on basis of a large amount of experimental and clinical data [62]. In general, choline is a dietary source of TMAO, but in a cohort study, there was no clear evidence of the association between choline intake and the risk of developing CVD [63]. Further, in ApoE (-/-) mice, L-carnitine administration led to resulted increased circulating TMAO levels but that was inversely related with atherosclerotic aortic plaque size [64]. Thus, the exact role of TMAO in atherosclerosis and CVD, as diagnostic tool and as having therapeutic potential is not fully established.

Secondary bile acids and atherosclerosis: The secondary bile acids are another group of gut microbiota-derived metabolites involved in CVD and various metabolic diseases [65]. Primary BAs are synthesized from cholesterol in the liver and stored in gall bladder and include cholic acid (CA) and chenodeoxycholic acid (CDCA). Primary BAs are usually metabolized into the secondary BAs, like deoxycholic acid (DCA) and lithocholic acid (LCA), hyodeoxycholic acid and ursodeoxycholic acid are formed from the primary Bas through gut microbiota-derived enzymes. There is a bidirectional relationship between gut microbiota and BA metabolism [66]. The hepatic BA biosynthesis influences the gut microbiota, highlighting the significance of the liver-BA-gut microbiome metabolic axis [67].

Both primary as well as secondary BAs are important signaling molecules that modulate the metabolism and energy expenditure. In addition to BAs, BA receptors mediate their biological functions and gut microbiota-derived secondary BAs play important roles in the development of atherosclerosis through the modulation of various BA receptors such as FXR, PXR, TGR5, VDR, and S1PR2 [68]. The BAs can promote the development of atherosclerosis mainly through bile-salt hydrolase (BSH) and BA receptors [69]. The C24 N-acyl bond of glycine-conjugated or taurine-conjugated bile salts are hydrolysed into free BAs by BSH. Further, the BA pool is chemically diversified by bacteria-derived $7 \alpha$-dehydroxylase and $7 \beta$-dehydroxylase to produce secondary Bas, which enter the portal circulation to function as signaling molecules and have impact on the homeostasis. Bacteria-mediated bile salt hydrolysis influences the processes underlying the pathogenesis of atherosclerosis by increasing cholesterol accumulation and foam cell formation [70]. The bile salt hydrolase is present in a variety of gut bacteria such as Methano brevibacter smithii, Clostridium, Enterococcus, etc [71].

\section{Gut Microbial Dysbiosis and Hypertension}

Apart from, dyslipidemia, hypertension is another most important risk factor for CVD that bears genetic susceptibility and influenced by environmental factors [72]. There exists a relation- ship between gut microbiota and hypertension, which has been evaluated in various recent studies. Simultaneously, the gut dysbiosis has been linked with cerebrovascular events [73].

It has long been established that antibiotic treatment can cause elevated blood pressure. The observation indirectly implicates the possible impact of the gut microbiota on regulation of blood pressure [74]. Further, in spontaneously hypertensive rats, it was documented that there occurs a significant decrease in gut microbial abundance and diversity and a relative increase in the ratio of Firmicutes/Bacteroidetes [75]. In another study in mice, the gut microbiota was shown to cause Ang-II-induced vascular dysfunction and hypertension [76]. Thus, it is apparent that the gut microbiota is involved in the development or exacerbation of hypertension. Though the exact underlying mechanisms and inter-relationship between gut microbiota and hypertension have not been established, the existing evidence from animal research and clinical studies highlights the roles of SCFAs and oxidized low-density lipoprotein (ox-LDL) in causation of hypertension.

\section{Short-chain fatty acids and hypertension}

The SCFAs, such as acetate, proprionate and butyrate are derived from soluble dietary fibre - mainly polysaccharides, in the intestine by the gut microbiota and play important role in maintaining homeostasis and immunity [77]. Further, the groups of gut microbes that metabolize polysaccharides into different types of SCFAs are specific. Thus, the major acetate-producing bacteria are Streptococcus, Prevotella, Bifidobacterium, Clostridiums and A. muciniphila [78]. Propionate is generated by Bacteroides, Salmonella, Dialister, Veillonella, Roseburia, Coprococcus, Blautia, etc [79]. Butyrate is produced by Lachnospiraceae, Ruminococcace, and Acid amino coccaceae [80]. The excess abundance of butyrate-producing bacteria has been associated with increased systolic and diastolic blood pressure in pregnant women [81]. Further, fibre and acetate supplementation led to an increase in Bacteroides acidifaciens and was associated with improvement of gut dysbiosis and hypertension and heart failure in hypertensive mice [82].

There are three G-protein-coupled receptors (GPCRs) which are regulated by SCFAs and include GPR41, GPR43, and GPR109A [83]. The SCFAs stimulate GPCRs-regulated pathways to influence renin-angiotensin system to modulate the blood pressure. Olfactory receptor 78 (Olfr78) is another type of GPCR expressed in the kidney, which is modulated by SCFAs [84]. Both Olfr78 and GPR41 are expressed in smooth muscle cells of small resistance blood vessels. In another study, the stimulation of GPR41 resulted in a reduction of the hypotensive response [85]. The SCFA, propionate induces vasodilation and produces an acute hypotensive response in mice through modulation of Olfr78 and GPR41 activity and this effect is opposed on stimulation of Olfr78 [86]. All these findings reveal that gut microbiota plays an important role in modulating the blood pressure through microbial SCFAs. The resultant hypertension related to dysbiosis, thus, can exacerbate CVD. 


\section{Oxidized low-density lipoprotein and hypertension}

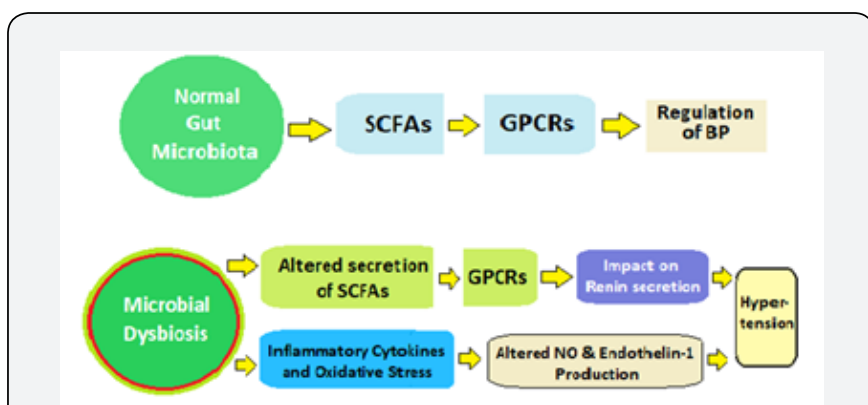

Figure 4: Gut microbiota's role in normal regulation of blood pressure and impact of dysbiosis.

The regulation of blood pressure, in general, depends on the net product of blood vessel vasoconstriction and vasodilation. In addition to the altered regulation of various receptors through SCFAs, gut dysbiosis also contributes to hypertension through vasoconstriction mediated by oxidation of LDL [87]. The microbial dysbiosis promotes expression of pro-inflammatory cytokines and induces oxidative stress, which stimulates Oxidation of LDL [88]. The higher levels of Ox-LDL decrease the production of NO and reduce the degree of vasodilation. Moreover, endothelin-1 plays crucial role in maintaining vascular tension and cardiovascular homeostasis. The activity of endothelin-1 on blood vessels is concentration-dependent, endothelin-1 produces vasodilatory effects at low concentrations by activating the endothelial receptor B and promoting NO production, and vasoconstriction at high concentrations by increasing ox-LDL production in plaques and activating the endothelial receptor A (Figure 4).

Still, the causative relationship between gut dysbiosis and hypertension is complex and has not been fully evaluated. The exact role of gut microbiota in mediating hypertension, the pathways and mechanisms involved require further elaborate research.

\section{Microbiota-Targeted Therapy for CVD}

The gut microbiota is a novel therapeutic target for the treatment of CVD, considering its involvement in the pathophysiology of CVD [89]. The imbalance in host-microbial interaction impairs homeostatic mechanisms that regulate health and activate multiple pathways leading to CV risk and CVD progression. The gut microbial dysbiosis contributes to the progression of CVD by promoting two major CVD risk factors, namely atherosclerosis and hypertension. These pathways contribute to the various stages of atherosclerotic plaque progression. In addition, the gut microbiome acts as an endocrine organ contributing several signaling molecules. These dysbiosis-generated metabolites promote hypertension through the alteration of vascular tone and vascular fibrosis.

\section{The prospects for MTT}

Gut microbiota-host interactions occur through various pathways, including the trimethylamine/trimethylamine $\mathrm{N}$-oxide pathway, short-chain fatty acids pathway, and primary and sec- ondary bile acids pathways. The presence of bacterial products in the systemic circulation and heightened inflammatory state contribute to further progression of atherosclerosis, altered CV homeostasis, hypertension and heart failure.

Over the recent years, there have evolved potential strategies for therapeutically targeting intestinal microbiota and microbial processes [90]. Further, the MTT, appears to be is a promising strategy to prevent and treat CVD [91,92]. The most frequently used measures to manipulate the gut microbiota to reduce $\mathrm{CV}$ risk and CVD are therapeutic use of probiotics, prebiotics and natural components, and intervention to repair microbial dysbiosis through fecal microbiota transplantation (FMT) $[93,94]$ (Figure 5).

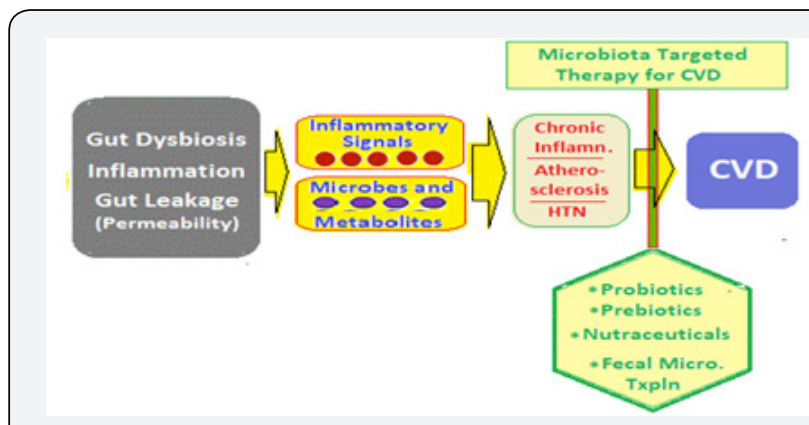

Figure 5: Various modalities of microbiota-based therapy for cardiovascular disease.

\section{The use of probiotics}

The commonly used probiotics are Lactobacillus, Bifidobacterium, and Satreptococcus. In a randomized double-blind clinical trial, Lactobacillus plantarum CECT 7527, 7528, and 7529 reduced circulating cholesterol levels and inhibited the formation of atherosclerotic plaques in hyper-cholesterol patients [95]. Lactobacillus reuteri NCIMB 30242 has also shown significant reductions of LDL-C and total cholesterol levels compared to placebo capsules [96].

The long-term administration of Lactobacillus bacteria (Lactobacillus fermentum CECT5716 (LC40), Lactobacillus coryniformis CECT5711 (K8) and Lactobacillus gasseri CECT5714 (LC9) have been shown to reduce systolic blood pressure [97]. L. plantarum ECGC13110402 is also well tolerated and can reduce cardiovascular risk [98].

\section{The use of prebiotics}

Prebiotic are compounds which can cause specific changes in the composition of gut microbiota and exert beneficial effects on host metabolism [99]. In studies in ApoE-/- mice, inulin-type fructans (ITFs) supplement improved endothelial function, while administration of ITFs promoted the production of butyrate and resulted in atherosclerosis-protective effects [100]. long-chain inulin also inhibits the formation of atherosclerotic plaque in ApoE/- mice, associated with alterations in lipid metabolism [101]. The consumption of $\beta$-glucan alters the composition of gut microbiota and reduces of CVD risk markers. The mannan oligosaccharide 
(MOS) supplement also modulate the composition of gut microbiota, lowering plasma cholesterol levels and improves atherosclerotic plaques in high cholesterol diet-fed mice [102].

\section{The use of nutrients}

In addition to probiotic and prebiotic, some natural active ingredients from herbs, like berberine have shown anti-atherosclerotic effect along with an increased abundance of Akkermansia in the gut in ApoE-/- mice [103].

Another complex nutrient resveratrol also has protective effect on several cardiovascular risk factors such as dyslipidemia and TMAO by modulating the gut microbiota and improving the integrity of the gut barrier [104]. Further, resveratrol attenuates TMAO-induced atherosclerosis by decreasing gut microbiota-mediated TMAO synthesis and increasing BA metabolism [105].

\section{FMT: intervention to repair gut dysbiosis}

Introducing the 'healthy' bacteria from healthy subjects into the gastrointestinal tract of patients with dysfunctional microbiota and suffering from gut dysbiosis and its fallouts is a novel and effective therapeutic strategy [106]. In a clinical study, the insulin sensitivity of recipients improved significantly after 6 weeks of FMT from lean normal donors to recipients with metabolic syndrome. Simultaneously, the FMT increased abundance of butyrate-producing bacteria [107].

Though, there is increasing acceptance for the therapeutic use of FMT, partially due to its perception as a 'natural' treatment and its relatively inexpensive implementation, the range of risks and benefits remains poorly defined because the published FMT experience is limited. Further, there are ethical considerations and regulations regarding the FMT as a 'new biologic drug' enforced in Canada and by the US Food and Drug Administration (FDA) [108] But, so far FMT is not regulated in West by the European Medicines Agency, or elsewhere within China or Australia. Further, there are fears of the infectious potential of the therapy, which have led researchers to explore the use of 'synthetic stool' products with defined bacterial populations to ameliorate such concerns [109].

In future, FMT can be a pauci-strain type or multi-strain type depending on the fecal microbiota analysis of the recipient. The suitable strains can be picked-up from donor fecal sample, grown in cultures and transplanted through an appropriate route. Depending on the recipients' microbiota diagnostic analysis, the FMT using suitable pauci-strains may be a promising development. Further, frozen sample FMT can be considered. The latter opens a new possibility of freezing fecal samples of a person in healthy state to be used later as autologous FMT in case of need, something like the concept of stem cells bank.

\section{References}

1. Qin J, Li R, Raes J, Arumugam M, Burgdorf KS, et al. (2010) A human gut microbial gene catalogue established by metagenomic sequencing. Nature 464: 59-65.
2. Tang WH, Kitai T, Hazen SL (2017) Gut microbiota in cardiovascular health and disease. Circ Res 120(1): 1183-1196.

3. Emoto T, Yamashita T, Kobayashi T, Sasaki N, Hirota Y, et al. (2017) Characterization of gut microbiota profiles in coronary artery disease patients using data mining analysis of terminal restriction fragment length polymorphism: gut microbiota could be a diagnostic marker of coronary artery disease. Heart Vessels 32(1): 39-46.

4. Ley RE, Turnbaugh PJ, Klein S, Gordon JI (2006) Microbial ecology: human gut microbes associated with obesity. Nature 444(7122): 10221023.

5. Henao-Mejia J, Elinav E, Jin C (2012) Inflammasome-mediated dysbiosis regulates progression of NAFLD and obesity. Nature 482: 179-185.

6. Pedersen HK, Gudmundsdottir V, Nielsen HB (2016) Human gut microbes impact host serum metabolome and insulin sensitivity. Nature 535: 376-381.

7. Khan MT, Nieuwdorp M, Backhed F (2014) Microbial modulation of insulin sensitivity. Cell Metab 20(5): 753-760.

8. Mouzaki M, Comelli EM, Arendt BM (2013) Intestinal microbiota in patients with non-alcoholic fatty liver disease. Hepatology 58(1): 120127.

9. Zhu L, Baker SS, Gill C, Liu W, Alkhouri R, et al. (2013) Characterization of gut microbiomes in non-alcoholic steatohepatitis (NASH) patients: a connection between endogenous alcohol and NASH. Hepatology 57(2): 601-609.

10. Gopalakrishnan V, Helmink BA, Spencer CN, Reuben A, Wargo JA, et al (2018) The influence of the gut microbiome on cancer, immunity, and cancer immunotherapy. Cancer Cell 33(4): 570-580.

11. Tilg H, Adolph TE, Gerner RR, Moschen AR (2018) The intestinal microbiota in colorectal cancer. Cancer Cell 33(6): 954-964.

12. Kamo T, Akazawa H, Suda W, Saga-Kamo A, Shimizu Y, et al. (2017) Dysbiosis and compositional alterations with aging in the gut microbiota of patients with heart failure. PLoS One 12(3): e0174099.

13. Lau K, Srivatsav V, Rizwan A, Nashed A, Liu R, et al. (2017) Bridging the gap between gut microbial dysbiosis and cardiovascular diseases. Nutrients 9(8): E859.

14. Ma J, Li H (2018) The Role of Gut Microbiota in Atherosclerosis and Hypertension. Front Pharmacol 9: 1082.

15. Bäckhed F, Ding H, Wang T, Hooper LV, Koh GY, et al. (2004) The gut microbiota as an environmental factor that regulates fat storage. Proc Natl Acad Sci USA 101(4): 15718-15723.

16. Turnbaugh PJ, Ley RE, Mahowald MA, Magrini V, Mardis ER, et al. (2006) An obesity-associated gut microbiome with increased capacity for energy harvest. Nature 444(7122): 1027-1031.

17. Samuel BS, Shaito A, Motoike T, Rey FE, Backhed F, et al. (2008) Effects of the gut microbiota on host adiposity are modulated by the shortchain fatty-acid binding G protein-coupled receptor, Gpr41. Proc Natl Acad Sci USA 105(43): 16767-16772.

18. Tolhurst G, Heffron H, Lam YS, Parker HE, Habib AM, et al. (2012) Short-chain fatty acids stimulate glucagon-like peptide- 1 secretion via the G-protein-coupled receptor FFAR2. Diabetes 61(2): 364-371.

19. Bäckhed F, Manchester JK, Semenkovich CF, Gordon JI (2007) Mechanisms underlying the resistance to diet-induced obesity in germ-free mice. Proc Natl Acad Sci USA 104(3): 979-984.

20. Ley RE, Bäckhed F, Turnbaugh P, Lozupone CA, Knight RD, et al. (2005) Obesity alters gut microbial ecology. Proc Natl Acad Sci USA 102: 11070-11075 


\section{Current Research in Diabetes \& Obesity Journal}

21. Cani PD, Amar J, Iglesias MA, Poggi M, Knauf C, et al. (2007) Metabolic endotoxemia initiates obesity and insulin resistance. Diabetes 56(7): 1761-1772.

22. de La Serre CB, Ellis CL, Lee J, Hartman AL, Rutledge JC, et al. (2010) Propensity to high-fat diet-induced obesity in rats is associated with changes in the gut microbiota and gut inflammation. Am J Physiol Gastrointest Liver Physiol 299(4): G440-G448.

23. Muccioli GG, Naslain D, Bäckhed F, Reigstad CS, Lambert DM, et al (2010) The endocannabinoid system links gut microbiota to adipogenesis. Mol Syst Biol 6: 392.

24. Cani PD, Bibiloni R, Knauf C, Waget A, Neyrinck AM, et al. (2008) Changes in gut microbiota control metabolic endotoxemia-induced inflammation in high-fat diet-induced obesity and diabetes in mice. Diabetes 57(6): 1470-1481.

25. Belzer C, de Vos WM (2012) Microbes inside--from diversity to function: the case of Akkermansia. ISME J 6(8): 1449-1458.

26. Wall R, Ross RP, Shanahan F, O’Mahony L, O Mahony C, et al. (2009) Metabolic activity of the enteric microbiota influences the fatty acid composition of murine and porcine liver and adipose tissues. Am J Clin Nutr 89(5): 1393-1401.

27. Creely SJ, McTernan PG, Kusminski CM, Fisher fM, Da Silva NF, et al. (2007) Lipopolysaccharide activates an innate immune system response in human adipose tissue in obesity and type 2 diabetes. Am J Physiol Endocrinol Metab 292(3): E740-E747.

28. Amar J, Serino M, Lange C, Chabo C, Iacovoni J, et al. (2011) Involvement of tissue bacteria in the onset of diabetes in humans: evidence for a concept. Diabetologia 54(12): 3055-3061.

29. Lewis K, Lutgendorff F, Phan V, Söderholm JD, Sherman PM, et al. (2010) Enhanced translocation of bacteria across metabolically stressed epithelia is reduced by butyrate. Inflamm Bowel Dis 16(7): 1138-1148.

30. Cope K, Risby T, Diehl AM (2000) Increased gastrointestinal ethanol production in obese mice: implications for fatty liver disease pathogenesis. Gastroenterology 119(5): 1340-1347.

31. Spruss A, Kanuri G, Wagnerberger S, Haub S, Bischoff SC, et al. (2009) Toll-like receptor 4 is involved in the development of fructose-induced hepatic steatosis in mice. Hepatology 50(4): 1094-1104.

32. Henao-Mejia J, Elinav E, Jin C, Hao L, Mehal WZ, et al. (2012) Inflammasome-mediated dysbiosis regulates progression of NAFLD and obesity. Nature 482(7384): 179-185.

33. Shanab AA, Scully P, Crosbie O, Buckley M, O Mahony L, et al. (2011) Small intestinal bacterial overgrowth in non-alcoholic steatohepatitis: association with toll-like receptor 4 expression and plasma levels of interleukin 8. Dig Dis Sci 56(5): 1524-1534.

34. Drosos I, Tavridou A, Kolios G (2015) New aspects on the metabolic role of intestinal microbiota in the development of atherosclerosis. Metabolism 64(4): 476-481.

35. Jie Z, Xia H, Zhong SL (2017) The gut microbiome in atherosclerotic cardiovascular disease. Nat Commun 8: 845

36. Kasahara K, Tanoue T, Yamashita T, Yodoi K, Matsumoto T, et al. (2017) Commensal bacteria at the crossroad between cholesterol homeostasis and chronic inflammation in atherosclerosis. J Lipid Res 58(3): 519528.

37. Karlsson FH, Fak F, Nookaew I, Tremaroli V, Fagerberg B, et al. (2012) Symptomatic atherosclerosis is associated with an altered gut metagenome. Nat Commun 3: 1245.

38. Li J, Lin S, Vanhoutte PM, Woo CW, Xu A (2016) Akkermansia muciniphila protects against atherosclerosis by preventing metabolic endotoxemia-induced inflammation in apoe-/- mice. Circulation 133(24): 2434-2446
39. Chen WY, Wang M, Zhang J, Barve SS, McClain CJ, et al. (2017) Acrolein disrupts tight junction proteins and causes endoplasmic reticulum stress-mediated epithelial cell death leading to intestinal barrier dysfunction and permeability. Am J Pathol 187(12): 2686-2697.

40. Gui T, Shimokado A, Sun Y (2012) Diverse roles of macrophages in atherosclerosis: from inflammatory biology to biomarker discovery. Mediat Inflamm 2012: 693083.

41. Harris K, Kassis A, Major G, Chou CJ (2012) Is the gut microbiota a new factor contributing to obesity and its metabolic disorders?. J Obes 2012: 879151.

42. Akira S, Takeda K (2004) Toll-like receptor signalling. Nat Rev Immunol 4: 499-511.

43. Xu H, Barnes GT, Yang Q Tan G, Yang D, et al. (2003) Chronic inflammation in fat plays a crucial role in the development of obesity-related insulin resistance. J Clin Investig 112(12): 1821-1830.

44. Edfeldt K, Swedenborg J, Hansson GK, Yan ZQ (2002) Expression of toll-like receptors in human atherosclerotic lesions: a possible pathway for plaque activation. Circulation 105(10): 1158-1161.

45. Barton GM, Kagan JC (2009) A cell biological view of Toll-like receptor function: regulation through compartmentalization. Nat Rev Immunol 9(8): 535-542.

46. Guzzo C, Ayer A, Basta S, Banfield BW, Gee K (2012) IL-27 enhances LPS-induced proinflammatory cytokine production via upregulation of TLR4 expression and signaling in human monocytes. J Immunol 188(2): 864-873.

47. Laman JD, Schoneveld AH, Moll FL, van Meurs M, Pasterkamp G, et al. (2002) Significance of peptidoglycan, a proinflammatory bacterial antigen in atherosclerotic arteries and its association with vulnerable plaques. Am J Cardiol 90(2): 119-123.

48. Kanno S, Nishio H, Tanaka T, et al. (2015) Activation of an innate immune receptor, Nod1, accelerates atherogenesis in Apoe-/- mice. J. Immunol 194(2): 773-780.

49. Li X, Shimizu Y, Kimura I (2017) Gut microbial metabolite short-chain fatty acids and obesity. Biosci Microbiota Food Health 36(4): 135-140.

50. Bergeron N, Williams PT, Lamendella R, Faghihnia N, Grube A, et al. (2016) Diets high in resistant starch increase plasma levels of trimethylamine-N-oxide, a gut microbiome metabolite associated with CVD risk. Br J Nutr 116(12): 2020-2029.

51. Brown JM, Hazen SL (2018) Microbial modulation of cardiovascular disease. Nat Rev Microbiol 16(3): 171-181.

52. Brown JM, Hazen SL (2015) The gut microbial endocrine organ: bacterially derived signals driving cardiometabolic diseases. Annu Rev Med 66: 343-359.

53. Wang Z, Tang WH, Buffa JA, Fu X, Britt EB, et al. (2014) Prognostic value of choline and betaine depends on intestinal microbiota-generated metabolite trimethylamine-N-oxide. Eur Heart J 35(14): 904-910.

54. Shih DM, Wang Z, Lee R, Meng Y, Che N, et al. (2015) Flavin containing monooxygenase 3 exerts broad effects on glucose and lipid metabolism and atherosclerosis. J Lipid Res 56(1): 22-37.

55. Warrier M, Shih DM, Burrows AC, Ferguson D, Gromovsky AD, et al (2015) The TMAO-generating enzyme flavin monooxygenase 3 is a central regulator of cholesterol balance. Cell Rep 10(3): 326-338.

56. Chen K, Zheng X, Feng M, Li D, Zhang H (2017) Gut microbiota-dependent metabolite trimethylamine n-oxide contributes to cardiac dysfunction in western diet-induced obese mice. Front Physiol 8: 139.

57. Guasch-Ferre M, Hu FB, Ruiz-Canela M, Bulló M, Toledo E, et al. (2017) Plasma metabolites from choline pathway and risk of cardiovascular disease in the PREDIMED (Prevention with Mediterranean Diet) Study. J Am Heart Assoc 6(11): e006524. 


\section{Current Research in Diabetes \& Obesity Journal}

58. Troseid M, Ueland T, Hov JR, Svardal A, Gregersen I, et al. (2015) Microbiota-dependent metabolite trimethylamine- $\mathrm{N}$-oxide is associated with disease severity and survival of patients with chronic heart failure. J Int Med 277(6): 717-726.

59. Senthong V, Li XS, Hudec T, Coughlin J, Wu Y, et al. (2016a) Plasma trimethylamine $\mathrm{N}$-Oxide, a gut microbe-generated phosphatidylcholine metabolite, is associated with atherosclerotic burden. J Am Coll Cardiol 67(22): 2620-2628.

60. Senthong V, Wang Z, Li XS, Fan Y, Wu Y, et al. (2016b) Intestinal microbiota-generated metabolite trimethylamine- $\mathrm{N}$-Oxide and 5-year mortality risk in stable coronary artery disease: the contributory role of intestinal microbiota in a COURAGE-like patient cohort. J Am Heart Assoc 5(6): e002816.

61. Zhu W, Gregory JC, Org E, Buffa JA, Gupta N, et al. (2016) Gut microbial metabolite TMAO enhances platelet hyperreactivity and thrombosis risk. Cell 165(1): 111-124.

62. Zheng Y, Li Y, Rimm EB, Hu FB, Albert CM, et al. (2016) Dietary phosphatidylcholine and risk of all-cause and cardiovascular-specific mortality among US women and men. Am J Clin Nutr 104(1): 173-180.

63. Nagata C, Wada K, Tamura T, Konishi K, Kawachi T, et al. (2015) Choline and betaine intakes are not associated with cardiovascular disease mortality risk in Japanese men and women. J Nutr 145(8): 1787-1792.

64. Collins HL, Drazul-Schrader D, Sulpizio AC, Koster PD, Williamson Y, et al. (2016) L-Carnitine intake and high trimethylamine $\mathrm{N}$-oxide plasma levels correlate with low aortic lesions in ApoE (-/-) transgenic mice expressing CETP. Atherosclerosis 244: 29-37.

65. Kuipers F, Bloks VW, Groen AK (2014) Beyond intestinal soap-bile acids in metabolic control. Nat Rev Endocrinol 10(8): 488-498

66. Jones ML, Martoni CJ, Ganopolsky JG, Labbé A, Prakash S (2014) The human microbiome and bile acid metabolism: dysbiosis, dysmetabolism, disease and intervention. Exp Opin Biol Ther 14(4): 467-482.

67. Zheng X, Huang F, Zhao A, Lei S, Zhang Y, et al. (2017) Bile acid is a significant host factor shaping the gut microbiome of diet-induced obese mice. BMC Biol 15(1): 120 .

68. Joyce SA, Gahan CG (2017) Disease-associated changes in bile acid profiles and links to altered gut microbiota. Dig Dis 35(3): 169-177.

69. Ridlon JM, Harris SC, Bhowmik S, Kang DJ, Hylemon PB (2016) Consequences of bile salt bio-transformations by intestinal bacteria. Gut Microbes 7(1): 22-39.

70. Hansson GK, Robertson AK, Soderberg-Naucler C (2006) Inflammation and atherosclerosis. Annu Rev Pathol 1: 297-329.

71. Tremaroli V, Backhed F (2012) Functional interactions between the gut microbiota and host metabolism. Nature 489(7415): 242-249.

72. Townsend MK, Aschard H, De Vivo I, Michels KB, Kraft P (2016) Genomics, telomere length, epigenetics, and metabolomics in the nurses health studies. Am J Public Health 106(9): 1663-1668.

73. Yamashiro K, Tanaka R, Urabe T, Ueno Y, Yamashiro Y, et al. (2017) Gut dysbiosis is associated with metabolism and systemic inflammation in patients with ischemic stroke. PLoS One 12(2): e0171521.

74. Honour J (1982) The possible involvement of intestinal bacteria in steroidal hypertension. Endocrinology 110(1): 285-287.

75. Yang T, Santisteban MM, Rodriguez V, Li E, Ahmari N, et al. (2015) Gut dysbiosis is linked to hypertension. Hypertension 65(6): 1331-1340.

76. Karbach SH, Schonfelder T, Brandao I, Wilms E, Hörmann N, et al. (2016) Gut microbiota promotes angiotensin ii-induced arterial hypertension and vascular dysfunction. J Am Heart Assoc 5(9): e003698.

77. Koh A, De Vadder F, Kovatcheva-Datchary P, Backhed F (2016) From dietary fiber to host physiology: short-chain fatty acids as key bacterial metabolites. Cell 165(6): 1332-1345.
78. Rey FE, Faith JJ, Bain J, Muehlbauer MJ, Stevens RD, et al. (2010) Dissecting the in vivo metabolic potential of two human gut acetogens. J Biol Chem 285(29): 22082-22090.

79. Louis P, Flint HJ (2017) Formation of propionate and butyrate by the human colonic microbiota. Environ Microbiol 19(1): 29-41.

80. Duncan SH, Barcenilla A, Stewart CS, Pryde SE, Flint HJ (2002) Acetate utilization and butyryl coenzyme A ( $\mathrm{CoA})$ : acetate-CoA transferase in butyrate-producing bacteria from the human large intestine. Appl Environ Microbiol 68(10): 5186-5190.

81. Gomez-Arango LF, Barrett HL, McIntyre HD, Callaway LK, Morrison M, et al. (2016) Increased systolic and diastolic blood pressure is associated with altered gut microbiota composition and butyrate production in early pregnancy. Hypertension 68(4): 974-981.

82. Marques FZ, Nelson E, Chu PY, Horlock D, Fiedler A, et al. (2017) High-fiber diet and acetate supplementation change the gut microbiota and prevent the development of hypertension and heart failure in hypertensive mice. Circulation 135(10): 964-977.

83. Tan JK, McKenzie C, Marino E, Macia L, Mackay CR (2017) Metabolite-sensing g protein-coupled receptors-facilitators of diet-related immune regulation. Annu Rev Immunol 35: 371-402.

84. Pluznick JL, Protzko RJ, Gevorgyan H, Peterlin Z, Sipos A, et al. (2013) Olfactory receptor responding to gut microbiota-derived signals plays a role in renin secretion and blood pressure regulation. Proc Natl Acad Sci U.S.A 110(11): 4410-4415.

85. Natarajan N, Hori D, Flavahan S, Steppan J, Flavahan NA, et al. (2016) Microbial short chain fatty acid metabolites lower blood pressure via endothelial G protein-coupled receptor 41. Physiol Genomics 48(11): 826-834.

86. Miyamoto J, Kasubuchi M, Nakajima A, Irie J, Itoh H, et al. (2016) The role of short-chain fatty acid on blood pressure regulation. Curr Opin Nephrol Hypertens 25(5): 379-383.

87. Packer CS, Rice AE, Johnson TC (2014) Oxidized low density lipoprotein (OX-LDL) induced arterial muscle contraction signaling mechanisms. Open Hyperten J 6: 20-26.

88. Peluso I, Morabito G, Urban L, Ioannone F, Serafini M (2012) Oxidative stress in atherosclerosis development: the central role of LDL and oxidative burst. Endocr Metab Immune Disord Drug Targets 12(4): 351360.

89. Koopen AM, Groen AK, Nieuwdorp M (2016) Human microbiome as therapeutic intervention target to reduce cardiovascular disease risk. Curr Opin Lipidol 27(6): 615-622.

90. Daliri EB, Lee BH, Oh DH (2017) Current perspectives on antihypertensive probiotics. Probiot Antimicrob Proteins 9(2): 91-101.

91. Anbazhagan AN, Priyamvada S, Priyadarshini M (2017) Gut microbiota in vascular disease: therapeutic target? Curr Vasc Pharmacol 15(14): 291-295.

92. Santisteban MM, Qi Y, Zubcevic J, Kim S, Yang T, et al. (2017) Hypertension-linked pathophysiological alterations in the gut. Circ Res 120(2): 312-323.

93. Ettinger G, MacDonald K, Reid G, Burton JP (2014) The influence of the human microbiome and probiotics on cardiovascular health. Gut Microbes 5(6): 719-728.

94. He M, Shi B (2017) Gut microbiota as a potential target of metabolic syndrome: the role of probiotics and prebiotics. Cell Biosci 7: 54 .

95. Fuentes MC, Lajo T, Carrion JM, Cune J (2013) Cholesterol-lowering efficacy of Lactobacillus plantarum CECT 7527, 7528 and 7529 in hypercholesterolaemic adults. Br J Nutr 109: 1866-1872.

96. Jones ML, Martoni CJ, Prakash S (2012) Cholesterol lowering and inhibition of sterol absorption by Lactobacillus reuteri NCIMB 30242: a randomized controlled trial. Eur J Clin Nutr 66(11): 1234-1241. 


\section{Current Research in Diabetes \& Obesity Journal}

97. Gomez-Guzman M, Toral M, Romero M, Jiménez R, Galindo P, et al. (2015) Antihypertensive effects of probiotics Lactobacillus strains in spontaneously hypertensive rats. Mol Nutr Food Res 59(11): 23262336.

98. Costabile A, Buttarazzi I, Kolida S, Quercia S, Baldini J, et al. (2017) An in vivo assessment of the cholesterol-lowering efficacy of Lactobacillus plantarum ECGC 13110402 in normal to mildly hypercholesterolaemic adults. PLoS One 12(12): e0187964.

99. Yoo JY, Kim SS (2016) Probiotics and prebiotics: present status and future perspectives on metabolic disorders. Nutrients 8(3): 173.

100. Catry E, Bindels LB, Tailleux A, Lestavel S, Neyrinck AM, et al. (2018) Targeting the gut microbiota with inulin-type fructans: preclinical demonstration of a novel approach in the management of end othelial dysfunction. Gut 67(2): 271-283

101. Rault-Nania MH, Gueux E, Demougeot C, Demigné C, Rock E, et al. (2006) Inulin attenuates atherosclerosis in apolipoprotein E-deficient mice. Br J Nutr 96(5): 840-844.

102. Hoving LR, Katiraei S, Heijink M, Pronk A, van der Wee-Pals L, et al. (2018) Dietary mannan oligosaccharides modulate gut microbiota, increase fecal bile acid excretion, and decrease plasma cholesterol and atherosclerosis development. Mol Nutr Food Res 62(10) e1700942.

103. Zhu L, Zhang D, Zhu H, Zhu J, Weng S, et al. (2018) Berberine treatment increases Akkermansia in the gut and improves high-fat di-

This work is licensed under Creative Commons Attribution 4.0 Licens

DOI: 10.19080/CRDOJ.2019.11.555805 et-induced atherosclerosis in Apoe (-/-) mice. Atherosclerosis 268: 117-126.

104. Bird JK, Raederstorff D, Weber P, Steinert RE (2017) Cardiovascular and anti-obesity effects of resveratrol mediated through the gut microbiota. Adv Nutr 8(6): 839-849.

105. Chen ML, Yi L, Zhang Y, Zhou X, Ran L et al. (2016) Resveratrol attenuates trimethylamine-N-Oxide (TMAO)-induced atherosclerosis by regulating TMAO synthesis and bile acid metabolism via remodeling of the gut microbiota. MBio 7(2): e02210-15.

106. Colman RJ, Rubin DT (2014) Fecal microbiota transplantation as therapy for inflammatory bowel disease: a systematic review and meta-analysis. J Crohns Colitis 8(12): 1569-1581.

107. Vrieze A, Van Nood E, Holleman F, Salojärvi J, Kootte RS, et al. (2012) Transfer of intestinal microbiota from lean donors increases insulin sensitivity in individuals with metabolic syndrome. Gastroenterology 143(4): 913-916.

108. (2014) Draft Guidance for Industry: Enforcement Policy Regarding Investigational New Drug Requirements for Use of Fecal Microbiota for Transplantation to Treat Clostridium difficile Infection Not Responsive to Standard Therapies. Silver Spring, MD: US Food and Drug Administration.

109. Petrof E, Gloor G, Vanner S, Weese SJ, Carter D, et al. (2013) Stool substitute transplant therapy for the eradication of Clostridium difficile infection: 'repopulating' the gut. Microbiome 1(1): 3.

Your next submission with Juniper Publishers
will reach you the below assets
- Quality Editorial service
- Swift Peer Review
- Reprints availability
- E-prints Service
- Manuscript Podcast for convenient understanding
- Global attainment for your research
- Manuscript accessibility in different formats
( Pdf, E-pub, Full Text, Audio)
- Unceasing customer service
Track the below URL for one-step submission
https://juniperpublishers.com/online-submission.php

